D. Katz and L. J. Ratliff, Jr.

Nagoya Math. J.

Vol. 103 (1986), 39-66

\title{
U-ESSENTIAL PRIME DIVISORS AND SEQUENCES OVER AN IDEAL
}

\author{
DANIEL KATZ AND LOUIS J. RATLIFF, JR.
}

\section{§1. Introduction}

All rings in this paper are assumed to be commutative with identity, and they will generally also be Noetherian.

In several recent papers the asymptotic theory of ideals in Noetherian rings has been introduced and developed. In this new theory the roles played in the standard theory by associated primes, $R$-sequences, classical grade, and Cohen-Macaulay rings are played by, respectively, asymptotic prime divisors, asymptotic sequences, asymptotic grade, and locally quasiunmixed Noetherian rings. And up to the present time it has been shown that quite a few results from the standard theory have a valid analogue in the asymptotic theory, and a number of interesting and useful new results concerning the asymptotic prime divisors of an ideal in a Noetherian ring have also been proved. In fact the analogy between the two theories is so good that a very useful (but not completely valid) working guide is: results from the standard theory should have a valid analogue in the asymptotic theory. And, although asymptotic sequences are coarser than $R$-sequences (for example, they behave nicely when passing to $R / z$ with $z$ a minimal prime ideal in $R$ ), the converse of this working guide has also proved useful.

However, in a number of problems it has turned out that the asymptotic theory is a little too coarse, so it seemed worthwhile to try to develop a new theory that behaved nicely when passing to $R / z$ with $z$ an arbitrary prime divisor of zero (rather than just a minimal prime divisor of zero). Such a theory would then be intermediate between the standard and asymptotic theories, and would thereby surmount some of the problems encountered in the asymptotic theory. One candidate for this new intermediate theory was developed in [7], where it was called the "essential"

Received August 29, 1984. 
theory. (The word "essential" was chosen because of the fact that if $R$ is a semi-local domain and $\mathscr{P} \subseteq \operatorname{Spec} R$, then $\cap\left\{R_{P} ; P \in \mathscr{P}\right\}$ is a finite $R$ module if and only if every essential prime divisor of a principal ideal in $R$ is contained in some $P \in \mathscr{P}$; thus the localizations at these primes are somewhat analogous to the essential valuations of a Krull domain (whose intersection is the Krull domain).) It was shown in [7] that the essential theory is a good candidate for this new intermediate theory, since most of the results from the other two theories that are concerned with prime divisors, sequences, and grade have a valid analogue in the new theory. However, the analogy breaks down in two important regards. First, the essential prime divisors of $I$ do not coincide with the asymptotic prime divisors of $I$ when $R$ is local and its completion has no imbedded prime divisors of zero. And, second, it was shown in [19] that many of the results concerning sequences over an ideal and the cograde of an ideal in the other two theories do not have a valid analogue in the essential theory. Thus this new essential theory falls short of being the desired intermediate theory.

Therefore, in the present paper, we present a new candidate for this intermediate theory, and call it the " $u$-essential" theory. (The name comes from the fact that the $u$-essential prime divisors of $I$ are the contractions to $R$ of the essential prime divisors of $(u)$ in the Rees ring of $R$ with respect to $I$.) In this new theory the two deficiencies in the essential theory mentioned in the preceding paragraph are repaired, and it turns out to be an excellent analogue of the standard and asymptotic theories in all regards. Also, to some extent it emcompasses both the asymptotic and essential theories, since the asymptotic and essential prime divisors of $I$ are also $u$-essential prime divisors of $I$.

In some preliminary applications of this new theory, the second author has shown that a prime ideal $P$ in a Noetherian ring $R$ has a primary ideal $q$ all of whose powers are primary if and only if there exists some ideal $I \subseteq P$ such that $P$ is the only $u$-essential prime divisor of $I$. Then, because of the results on $u$-essential prime divisors established in (2.5), this leads to several other such primary ideals, both in $R$ and in certain rings related to $R$. Also, $u$-essential prime divisors have yielded some new results concerning $\operatorname{Ker}\left(R\left[X_{1}, \cdots, X_{n}\right] \rightarrow R\left[b_{1} / b_{0}, \cdots, b_{n} / b_{0}\right]\right)$ and also the ring $R^{(1)}=\cap\left\{R_{P}\right.$; height $\left.P=1\right\}$. Thus this new theory seems to be very useful in surmounting some of the problems encountered when using 
the asymptotic theory, so we thought it would be desirable to have a paper where the basics of the $u$-essential theory are developed-and this is the purpose of the present paper.

In Section 2 we develop quite a few of the basic properties of $u$ essential prime divisors. In particular, it is shown that they behave nicely when passing to localizations, factor rings modulo prime divisors of zero, faithfully flat Noetherian extension rings, and finite integral extension rings.

In Section 3 it is shown that $u$-essential sequences over an ideal $I$ also behave nicely when passing to the same type of related rings, and in Section 4 it is shown that this also holds for the $u$-essential cograde of $I$. In Section 5 several preliminary results for Section 6 are proved, and in Section 6 it is shown that most of the bounds on the asymptotic cograde of $I$ given in [6] have a valid analogue for the $u$-essential cograde. Finally, in Section 7 we give several examples to show some of the differences between essential sequences over $I$ and $u$-essential sequences over $I$.

As already mentioned, the results in this paper are closely analogous to the previously developed asymptotic theory. They are meant to present a new intermediate theory between the standard and asymptotic theories, and we feel these results show that the $u$-essential theory is the natural choice for such an intermediate theory. The applications of this new theory to date have been very promising, and we think this new theory will have many important applications in future work on the ideal theory of Noetherian rings.

We are indebted to the referee for his suggestions on simplifying several of our proofs and for correcting our original proofs of (5.1) and (7.2).

\section{§2. U-essential prime divisors}

In this section we prove a number of properties of the $u$-essential prime divisors of an ideal $I$ in a Noetherian ring $R$. (The name comes from their definition: they are the contraction to $R$ of the essential prime divisors of $u$ in the Rees ring of $R$ with respect to $I$.) These prime ideals were first considered in [3], and a few of their basic properties were established there. In this section we give a more complete study of these ideals. We begin with the basic definitions. 
All rings in this paper are commutative with identity and they will generally be Noetherian. If $R$ is a semi-local (Noetherian) ring, then $R^{*}$ will denote the completion of $R$ in its natural topology. And if $I$ is an ideal in a Noetherian ring $R$, then $\mathscr{R}=\mathscr{R}(R, I)$ will denote the Rees ring of $R$ with respect to $I$; that is, $\mathscr{R}=R[u, t I]$, where $t$ is an indeterminate and $u=1 / t$. Thus $\mathscr{R}$ is a graded Noetherian subring of $R[u, t], u$ is a regular element in $\mathscr{R}$, and $u^{n} \mathscr{R} \cap R=I^{n}$ for all $n \geqslant 1$.

(2.1) Definition. Let $I$ be an ideal in a Noetherian ring $R$ and let $b_{1}, \cdots, b_{d}$ be nonunits in $R$. Then:

(2.1.1) $A^{*}(I)=\left\{P \in \operatorname{Spec} R ; P \in\right.$ Ass $R / I^{n}$ for all large $\left.n\right\}, \hat{A}^{*}(I)=\{P \in$ Spec $R ; P \in$ Ass $R /\left(I^{n}\right)_{a}$ for all large $\left.n\right\}$, where $\left(I^{n}\right)_{a}$ is the integral closure in $R$ of $I^{n}, E(I)=\left\{P \in \operatorname{Spec} R ; I\left(R_{P}\right)^{*}+z\right.$ is $P\left(R_{P}\right)^{*}$-primary for some $\left.z \in \operatorname{Ass}\left(R_{P}\right)^{*}\right\}$, and, $U(I)=\{p \cap R ; p \in E(u \mathscr{R}(R, I))\} . \quad P$ is an asymptotic (resp., essential, u-essential) prime divisor of $I$ in case $P \in \hat{A}^{*}(I)$ (resp., $P \in E(I), P \in U(I))$.

(2.12) $b_{1}, \cdots, b_{d}$ are an asymptotic (resp., essential, u-essential) sequence over $I$ in case $\left(I, b_{1}, \cdots, b_{d}\right) R \neq R$ and $b_{i} \notin \cup \hat{A}^{*}\left(\left(I, b_{1}, \cdots, b_{i-1}\right) R\right.$ ) (resp., $\left.\left.b_{i} \notin \cup E\left(\left(I, b_{1}, \cdots, b_{i-1}\right) R\right), b_{i} \notin \cup U\left(I, b_{1}, \cdots, b_{i-1}\right) R\right)\right)$ for $i=1, \cdots, d$. An asymptotic (resp., essential, $u$-essential) sequence over (0) is simply called an asymptotic (resp., essential, u-essential) sequence in $R$. (It is shown in (3.10) that $b_{1}, \cdots, b_{a}$ are a $u$-essential sequence in $R$ if and only if they are an essential sequence in $R$, so the terminology " $u$-essential sequence in $R$ " will only be used till (3.10) is proved.)

(2.1.3) The asymptotic (resp., essential) grade of $I$, denoted agd $(I)$ (resp., egd $(I)$ ) is the length of an asymptotic (resp., essential) sequence maximal with respect to coming from $I$.

(2.1.4) If $R$ is local, then the asymptotic (resp., essential, u-essential) cograde of $I$, denoted acogd $(I)$ (resp., ecogd $(I)$, uecogd $(I)$ ), is the length of a maximal asymptotic (resp., essential, $u$-essential) sequence over $I$.

The concepts of a $u$-essential sequence over $I$ and of uecogd $(I)$ are new to this paper. But the other concepts defined in (2.1) have previously been studied and a number of their properties have been determined. In what follows we will need to use several of these properties, so (2.2) contains a list of those that are most often used below.

(2.2) Remark. Let $I$ be an ideal in a Noetherian ring $R$. Then the following hold: 
(2.2.1) The sets Ass $R / I^{n}$ and Ass $R /\left(I^{n}\right)_{a}$ are stable for all large $n$, by [1] and [17, (2.7)] (see also [8]), so $A^{*}(I)$ and $\hat{A}^{*}(I)$ are well defined finite sets of prime ideals. Also, $\hat{A}^{*}(I) \subseteq A^{*}(I)$ and $E(I) \subseteq A^{*}(I)$, by [17, (2.7)] and [7, (3.3.1)], so $E(I)$ is also a finite set of prime divisors of $I^{n}$ for all large $n$.

(2.2.2) It is clear from the definitions that each minimal prime divisor of $I$ is in $\hat{A}^{*}(I) \cap E(I)$.

(2.2.3) If $z \in$ Ass $R$ and $P$ is a minimal prime divisor of $I+z$, then $P \in E(I)$, by [7, (3.3.4)].

(2.2.4) If $P \in \operatorname{Spec} R$ and $S$ is a multiplicatively closed set in $R$ such that $P_{S} \neq R_{S}$, then $P \in \hat{A}^{*}(I)$ (resp., $E(I)$ ) if and only if $P_{S} \in \hat{A}^{*}\left(I_{S}\right)$ (resp., $E\left(I_{S}\right)$ ), by [18, (2.9.2)] (resp., [7, (3.3.2)]).

(2.2.5) $P \in \hat{A}^{*}(I)$ (resp., $E(I)$ ) if and only if $P / z \in \hat{A}^{*}((I+z) / z$ ) (resp., $E((I+z) / z)$ ) for some minimal (resp., for some) $z \in$ Ass $R$, by [17, (6.3)] (resp., [7, (3.6)]).

(2.2.6) If $A$ is a Noetherian ring which is a faithfully flat $R$-module, then $\hat{A}^{*}(I)=\hat{A}^{*}(I A) \cap R$ (resp., $E(I)=E(I A) \cap R$ ), and if $P \in \hat{A}^{*}(I)$ (resp., $E(I)$ ) and $P^{*}$ is a minimal prime divisor of $P A$, then $P^{*} \in \hat{A}^{*}(I A)$ (resp., $E(I A)$ ), by [17, (6.5) and (6.8)] (resp., [7, (3.7)]).

(2.2.7) If $B$ is a finite integral extension ring of $R$, then $\hat{A}^{*}(I) \subseteq$ $\hat{A}^{*}(I B) \cap R$ (resp., $\left.E(I) \subseteq E(I B) \cap R\right)$. Moreover, if $z \in$ Ass $B$ implies $z \cap R \in$ Ass $R$, then equality holds, by [20] (resp., [7, (3.9)]).

(2.2.8) If $J$ is an ideal in $R$ such that $\operatorname{Rad} J=\operatorname{Rad} I$, then $E(J)=$ $E(I)$, by [7, (3.3.5)].

(2.2.9) It follows immediately from (2.1.2) that if $b_{1}, \cdots, b_{d}$ in $R$ are an asymptotic (resp., essential) sequence over $I$, then height $\left(I, b_{1}, \cdots, b_{d}\right) R \geqslant$ height $I+d$. Therefore, by the Generalized Principal Ideal Theorem, if $b_{1}, \cdots, b_{d}$ are an asymptotic (resp., essential) sequence in $R$, then height $\left(b_{1}, \cdots, b_{d}\right) R=d$.

(2.2.10) If $b_{1}, \cdots, b_{d}$ in $R$ are an asymptotic (resp., essential) sequence over $I$ and $S$ is a multiplicatively closed set in $R$ such that $\left(I, b_{1}, \cdots, b_{d}\right)$ $\cdot R_{s} \neq R_{s}$, then the images of $b_{1}, \cdots, b_{d}$ in $R_{s}$ are an asymptotic (resp., essential) sequence over $I_{S}$, by [18, (2.9.2)] (resp. [19, (2.3)]).

(2.2.11) Elements $b_{1}, \cdots, b_{d}$ in $R$ are an asymptotic (resp., essential) sequence over $I$ if and only if their images in $R / z$ are an asymptotic (resp., essential) sequence over $(I+z) / z$ for all minimal (resp., for all) $z \in$ Ass $R$, by [17, (6.3)] (resp., [19, (2.4)]). 
(2.2.12) If $A$ is a Noetherian ring which is a faithfully flat $R$-module, then $b_{1}, \cdots, b_{d}$ in $R$ are an asymptotic (resp., essential) sequence over $I$ if and only if they are an asymptotic (resp., essential) sequence over $I A$, by $[17,(6.5)$ and (6.8)] (resp., [19, (2.5)]).

(2.2.13) Agd $(I)$ (resp., egd $(I)$ ) is unambiguously defined and agd $(I)$ (resp., egd $(I))=\min \left\{\right.$ height $\left(I\left(R_{P}\right)^{*}+z\right) / z ; I \subseteq P \in \operatorname{Spec} R$ and $z$ is minimal in Ass $\left(R_{P}\right)$ (resp., $\left.\left.z \in \operatorname{Ass}\left(R_{P}\right)^{*}\right)\right\}$, by [18, (3.1)] (resp., [19, (5.3)]).

(2.2.14) If $R$ is local, then $\operatorname{acogd}(I)$ (resp., ecogd $(I)$ ) is unambiguously defined and $\operatorname{acogd}(I)=\min \left\{\operatorname{depth} z-\ell\left(\left(I R^{*}+z\right) / z\right) ; z\right.$ is minimal in Ass $\left.R^{*}\right\}$ (resp., ecogd $(I)=\min \left\{\operatorname{depth}\left(I R^{*}+z\right) ; z \in\right.$ Ass $\left.R^{*}\right\}$ ), by [2] (resp., $[19,(3.2)])$. Here $\ell(J)$ denotes the analytic spread of the ideal $J$.

We now begin considering $u$-essential prime divisors. Our first result, (2.3), contains three of their basic properties.

(2.3) Remark. If $I$ is an ideal in a Noetherian ring $R$, then the following hold:

(2.3.1) $U((0))=$ Ass $R$.

(2.3.2) If $P$ is a minimal prime divisor of $I$, then $P \in U(I)$.

(2.3.3) $U(I) \subseteq A^{*}(I)$.

Proof. Note that $\mathscr{R}=\mathscr{R}(R,(0))=R[u]$ and $u$ is an indeterminate. Therefore $E(u \mathscr{R})=E(u R[u])=\left\{(z, u) R[u] ; z \in E\left(\left(0_{R}\right)\right)\right\}$, by [19, (2.7)], and $E\left(\left(0_{R}\right)\right)=$ Ass $R$, by [7, (3.3.3)], so $U((0))=\{(z, u) \mathscr{R} \cap R ; z \in$ Ass $R\}=$ Ass $R$. This proves (2.3.1).

For (2.3.2) let $\mathscr{R}=\mathscr{R}(R, I)$. Then $u \mathscr{R} \cap R=I$, so if $P$ is a minimal prime divisor of $I$, then there exists a minimal prime divisor $p$ of $u \mathscr{R}$ such that $p \cap R=P$, and $p \in E(u \mathscr{R})$, by (2.2.2), so $P \in U(I)$.

For (2.3.3), let $\mathscr{R}=\mathscr{R}(R, I)$. Then it was shown in [4, Corollary 17] that if $p$ is a prime divisor of $u \mathscr{R}$ such that $t I \not p$, then $p \cap R \in A^{*}(I)$. And in [16, Corollary 3.16] it was shown that if $I \subseteq P \in$ Ass $R$, then $P \in A^{*}(I)$. Therefore it suffices to show that if $p \in E(u \mathscr{R})$ and $P=p \cap R$, then either $P \in$ Ass $R$ or $t I \not \subset p$. For this, assume that $P \notin$ Ass $R$ and suppose that $t I \subseteq p$. Let $S=R-P$. Then $\mathscr{R}\left(R_{P}, I_{P}\right)=\mathscr{R}_{S}, t I_{P} \subseteq p \mathscr{R}_{S}$, and $p \mathscr{R}_{S} \in E\left(u \mathscr{R}_{S}\right)$, by (2.2.4). Also $P_{P} \notin$ Ass $R_{P}$, so it may be assumed that $R$ is local with maximal ideal $P$. Let $\mathscr{S}=\mathscr{R}\left(R^{*}, I R^{*}\right)$. Now $p$ is the maximal homogeneous ideal in $\mathscr{R}$, since $(u, P, t I) \mathscr{R} \subseteq p$, so $p \mathscr{S}$ is the maximal homogeneous ideal in $\mathscr{S}$ and $\mathscr{R}_{p}$ is a dense subspace of $\mathscr{S}_{p \mathscr{S}}$, by [12, Lemma 3.2]. Let $L=\mathscr{S}_{p \varphi}$, so $L^{*}=\left(\mathscr{R}_{p}\right)^{*}$, and so there exists $z^{*} \in \operatorname{Ass} L^{*}$ 
such that $\left(z^{*}, u\right) L^{*}$ is $p L^{*}$-primary, since $p \in E(u \mathscr{R})$. Let $z=z^{*} \cap \mathscr{S}$ and $w=z \cap R^{*}$, so $z \in \operatorname{Ass} \mathscr{S}$, and so $w \in$ Ass $R^{*}$ and $\mathscr{S} / z \cong \mathscr{R}\left(R^{*} / w\right.$, (IR* + $w) / w$ ), by [22, Theorem 1.5 and Lemma 1.1]. Now $p \mathscr{S} / z$ is a maximal ideal in $\mathscr{S} / z$ and $\mathscr{S} / p \mathscr{S}=R^{*} / P R^{*}$, and $\operatorname{trd}(\mathscr{S} / z) /\left(R^{*} / w\right)=1$, by the isomorphism, so height $p \mathscr{S} / z+0=$ height $P R^{*} / w+1$, since $R^{*} / w$ satisfies the altitude formula. Therefore, since $P \notin$ Ass $R$, it follows that height $P R^{*} / w \geqslant 1$, so height $p \mathscr{S} / z \geqslant 2$. But $L / z L$ is unmixed and analytically unramified, by [15, (6.5)], and $z^{*}$ is a prime divisor of $z L^{*}$, so necessarily $z^{*} / z L^{*}$ is a minimal prime ideal and $\left(z^{*}, u\right) L^{*} / z L^{*}$ is $p L^{*} / z L^{*}$-primary. Hence height $p L^{*} / z L^{*}$ $=1$, and so height $p \mathscr{S} \mid z=1$, and this is a contradiction. Therefore $t I \not \subset p$, so $U(I) \subseteq A^{*}(I)$.

q.e.d.

Before proving the main result in this section, (2.5), we need the following lemma which shows that in an important special case the asymptotic, essential, and $u$-essential prime divisors of certain ideals are all the same.

(2.4) Lemma. Let $R$ be a locally unmixed Noetherian ring, let $b_{1}, \cdots, b_{d}$ be elements in $R$ such that height $\left(b_{1}, \cdots, b_{i}\right) R=i$ for $i=1, \cdots, d$, and let $B=\left(b_{1}, \cdots, b_{d}\right) R$. Then $b_{1}, \cdots, b_{d}$ are an essential sequence in $R$ and $\hat{A}^{*}(B)=E(B)=U(B)=\{P \in \operatorname{Spec} R ; P$ is a minimal prime divisor of $B$.

Proof. It is shown in [7, (6.1)] that $b_{1}, \cdots, b_{d}$ are an essential sequence in $R$ and that $E(B)=\mathscr{P}$, where $\mathscr{P}=\{P \in \operatorname{Spec} R ; P$ is a minimal prime divisor of $B$. Also, since $R$ is also locally quasi-unmixed and $B$ is an ideal of the principal class, $\hat{A}^{*}(B)=\mathscr{P}$ (by [13, Theorem 2.12]).

Now let $P \in U(B)$ and let $p \in E(u \mathscr{R})$ such that $p \cap R=P$, where $\mathscr{R}=$ $\mathscr{R}(R, B)$. Then it follows from [9, Corollary, p. 61] that $\mathscr{R}$ is locally unmixed, so height $p=1$, by [7, (6.1)]. Let $z^{*} \in$ Ass $\mathscr{R}$ such that $z^{*} \subset p$ and let $z=z^{*} \cap R$. Then $z \in$ Ass $R$ and $\mathscr{R} / z^{*} \cong \mathscr{R}(R / z,(B+z) / z)$, by [22, Theorem 1.5 and Lemma 1.1], and $R / z$ satisfies the altitude formula, by [12, Corollary 2.7]. Therefore height $p / z^{*}+\operatorname{trd}(\mathscr{R} / p) /(R / P)=$ height $P / z+$ $\operatorname{trd}\left(\mathscr{R} / z^{*}\right) /(R / z)$; that is, $t=\operatorname{trd}(\mathscr{R} / p) /(R / P)=$ height $P / z$. But $t \leqslant d$ (since $u \in p$ and $\mathscr{R}$ is generated by $u, t b_{1}, \cdots, t b_{d}$ over $R$ ), and height $P / z \geqslant d$, by (2.2.9) (since the images of $b_{1}, \cdots, b_{d}$ in $R / z$ are an essential sequence, by (2.2.11)). Therefore height $P / z=d$. Now $R_{P}$ satisfies th 3 first chain condition for prime ideals, since $R_{P}$ is unmixed, so it follows that height $P$ $=d$, so $P \in \mathscr{P}$, hence $U(B) \subseteq \mathscr{P}$. Finally, if $P \in \mathscr{P}$, then there exists a 
minimal prime divisor $p$ of $u \mathscr{R}$ such that $p \cap R=P$, since $u \mathscr{R} \cap R=B$, so $p \in E(u \mathscr{R})$, by (2.2.2), hence $P \in U(B)$, and so $U(B)=\mathscr{P}$. q.e.d.

The following internal characterization of $U(I)$ was given in [3, Theorem 2.5]: $\quad U(I)=\bigcap\left\{A^{*}(J)\right.$; $J$ is an ideal in $R$ and $I^{n} \subseteq J \subseteq\left(I^{n}\right)_{a}$ for some $n \geqslant 1$. But even with this characterization the $u$-essential primes are somewhat awkward to work with. (2.5) shows that $U(I)$ behaves very nicely with respect to passing to certain related rings. This is important, since they are very useful ideals, and once (2.5) is proved they will be considerably less awkward to work with.

(2.5) Theorem. Let $I$ be an ideal in a Noetherian ring $R$. Then the following hold:

(2.5.1) If $S$ is a multiplicatively closed set in $R$, then $U\left(I_{S}\right)=\left\{P_{S}\right.$; $P \in U(I)$ and $P \cap S=\phi\}$.

(2.5.2) $P \in U(I)$ if and only if there exists $z \in$ Ass $R$ such that $z \subseteq P$ and $P / z \in U((I+z) / z)$.

(2.5.3) If $A$ is a Noetherian ring which is a faithfully flat $R$-module, then $U(I)=\left\{P^{*} \cap R ; P^{*} \in U(I A)\right\}$, and if $P \in U(I)$ and $P^{*}$ is a minimal prime divisor of $P A$, then $P^{*} \in U(I A)$.

(2.5.4) If $B$ is a finite integral extension ring of $R$, then $U(I) \subseteq$ $\left\{P^{\prime} \cap R ; P^{\prime} \in U(I B)\right\}$, and equality holds if $z \in$ Ass $B$ implies $z \cap R \in$ Ass $R$.

(2.5.5) $U((I, X) R[X])=\{(P, X) R[X] ; P \in U(I)\}$.

(2.5.6) If $J$ is an ideal in $R$ that is projectively equivalent to $I$, that $i s$, there exist positive integers $m$ and $n$ such that $\left(I^{n}\right)_{a}=\left(J^{m}\right)_{a}$, then $U(J)$ $=U(I)$.

(2.5.7) $\hat{A}^{*}(I) \cup E(I) \subseteq U(I)$.

(2.5.8) If $I$ is generated by an essential sequence in $R$, then $U(I)=E(I)$.

Proof. Throughout, $\mathscr{R}=\mathscr{R}(R, I)$.

For (2.5.1) let $Q \in U\left(I_{S}\right)$ and let $q \in E\left(u \mathscr{R}\left(R_{S}, I_{S}\right)\right)$ such that $q \cap R_{S}=Q$. Then $\mathscr{R}\left(R_{S}, I_{S}\right)=\mathscr{R}_{S}$, so $p=q \cap \mathscr{R} \in E(u \mathscr{R})$, by (2.2.4), hence $P=p \cap R \in$ $U(I)$, by (2.1.1), and $Q=P_{S}$. Conversely, if $P \in U(I)$ and $P \cap S=\phi$, then let $p \in E(u \mathscr{R})$ such that $p \cap R=P$. Then $p_{S} \in E\left(u \mathscr{R}_{S}\right)$, by (2.2.4), and $p_{S} \cap R_{S}=P_{S}$, so $P_{S} \in U\left(I_{S}\right)$, by (2.1.1).

For (2.5.2) let $T$ be the total quotient ring of $\mathscr{R}$. Then Ass $\mathscr{R}=$ $\{z T \cap \mathscr{R} ; z \in \operatorname{Ass} R\}$, by [22, Theorem 1.5], and if $z \in$ Ass $R$, then $\mathscr{R} /(z T \cap \mathscr{R})$ $\cong \mathscr{R}(R / z,(I+z) / z)$, by [22, Lemma 1.1]. Therefore (2.5.2) follows readily from (2.2.5) (applied to $u \mathscr{R}$ ) and (2.1.1). 
For (2.5.3), let $\mathscr{A}=\mathscr{R}(A, I A)$, so $\mathscr{A}$ is a faithfully flat $\mathscr{R}$-module, by [17, (6.4)]. Therefore, if $P \in U(I)$, then let $p \in E(u \mathscr{R})$ such that $p \cap R=P$ and let $p^{*}$ be a minimal prime divisor of $p \mathscr{A}$. Then $p^{*} \in E(u \mathscr{A})$, by (2.2.6), so $P^{*}=p^{*} \cap A \in U(I A)$, by (2.1.1), and $P^{*} \cap R=P$. Therefore $U(I) \subseteq$ $\left\{P^{*} \cap R ; P^{*} \in U(I A)\right\}$, and the proof of the opposite inclusion is similar. Finally, let $P \in U(I)$ and let $P^{*}$ be a minimal prime divisor of $P A$. Then $R_{P} \subseteq A_{P^{*}}$ satisfy the Theorem of Transition. Therefore, since $P_{P} \in U\left(I_{P}\right)$, by (2.5.1), it follows from what has already been proved that there exists $Q^{*} \in U\left(I A_{P^{*}}\right)$ that lies over $P_{P}$, so $Q^{*}=P_{P^{*}}^{*}$, hence $P^{*} \in U(I A)$, by (2.5.1).

For (2.5.4), let $\mathscr{B}=\mathscr{R}(B, I B)$, so $\mathscr{B}$ is a finite integral extension ring of $\mathscr{R}$. Therefore the set containment follows readily from (2.2.7) (applied to $u \mathscr{R})$ and (2.1.1). Also, the prime divisor of zero in $\mathscr{R}$ are the ideals $z T \cap \mathscr{R}$ with $z \in$ Ass $R$ and $T$ the total quotient ring of $\mathscr{R}$, by [22, Theorem 1.5], and a similar statement holds for $\mathscr{B}$. Therefore the last statement in (2.5.4) also readily follows from (2.2.7) (applied to $u \mathscr{R}$ ) and (2.1.1).

For (2.5.5) let $P \in U(I)$. Then by (2.5.1), $P_{P} \in U\left(I_{P}\right)$ and if $\left(P_{P}, X\right) R_{P}[X] \in$ $U\left(\left(I_{P}, X\right) R_{P}[X]\right)$, then $(P, X) R[X] \in U((I, X) R[X])$, so it may be assumed that $R$ is local with maximal ideal $P$. Then similarly by using (2.5.3) and (2.5.2) it may be assumed that $R$ is a complete local domain with maximal ideal $P$. Let $\mathscr{R}=\mathscr{R}(R, I)$ and let $p \in E(u \mathscr{R})$ such that $p \cap R=P$. Then $q=(p, X) \mathscr{R}[X] \in E((u, X) \mathscr{R}[X])$, by $[19,(2.7)]$. Now $\mathscr{R}[X]$ is locally unmixed, by [9, Corollary, p. 61], so height $q=2$, by (2.4). Also, $X / u=t X$, so $q^{\prime}=q \mathscr{R}[X, t X]$ is a height one prime ideal that contains $u \mathscr{R}[X, t X]$ and lies over $q$, by [14, Lemma 2.7]. Therefore $q^{\prime} \in E(u \mathscr{R}[X, t X])$, by (2.2.2), and $\mathscr{R}[X, t X]=\mathscr{R}(R[X],(I, X), R[X])$, so $(P, X) R[X]=q^{\prime} \cap R[X] \in U((I, X) R[X])$.

Now let $Q \in U((I, X) R[X])$ and let $P=Q \cap R$, so $Q=(P, X) R[X]$. As in the preceding paragraph it may be assumed that $R$ is a complete local domain with maximal ideal $P$. Therefore let $\mathscr{R}=\mathscr{R}(R, I)$ and, since $\mathscr{R}(R[X],(I, X) R[X])=\mathscr{R}[X, t X]=\left(\right.$ say) $\mathscr{S}$, let $q^{\prime} \in E(u \mathscr{S})$ such that $q^{\prime} \cap$ $R[X]=Q$. Then height $q^{\prime}=1$, by (2.4), and $(u, X) \mathscr{R}[X] \subseteq q=q^{\prime} \cap \mathscr{R}[X]$, so by the altitude formula (since $R$ is a complete local domain) it follows that height $q=2$. Therefore $q \in E((u, X) \mathscr{R}[X])$, by (2.4), and so [19, (2.7)] implies that $q=(p, X) \mathscr{R}[X]$ where $p=q \cap \mathscr{R} \in E(u \mathscr{R})$. Finally, $p \cap R=P$, so $P \in U(I)$.

For (2.5.6), let $\mathscr{S}=\mathscr{R}\left(R, I_{a}\right)$, so $\mathscr{S}$ is a finite integral extension ring of $\mathscr{R}$ (since $I$ reduces $I_{a}$ ) and $\mathscr{R}$ and $\mathscr{S}$ have the same total quotient ring. 
Therefore (2.2.7) implies $E(u \mathscr{R})=E(u \mathscr{S}) \cap \mathscr{R}$. Thus it follows that $U\left(I_{a}\right)=U(I)$.

Now let $\mathscr{A}=R\left[u^{n}, t^{n} I^{n}\right]$. Then $\mathscr{R}$ is a finite integral extension ring of $\mathscr{A}$ and $\mathscr{A} \cong \mathscr{R}\left(R, I^{n}\right)$. Also, it follows from the description of Ass $\mathscr{R}$ given in the proof of (2.5.2) that $z \in$ Ass $\mathscr{R}$ implies $z \cap \mathscr{A} \in$ Ass $\mathscr{A}$. Therefore, since $E(u \mathscr{R})=E\left(u^{n} \mathscr{R}\right)$, by (2.2.8), it follows from (2.2.7) that $E\left(u^{n} \mathscr{A}\right)=$ $E(u \mathscr{R}) \cap \mathscr{A}$. Therefore it follows that $U(I)=U\left(I^{n}\right)$ and, similarly, that $U(J)=U\left(J^{m}\right)$. Also, $U\left(I^{n}\right)=U\left(\left(I^{n}\right)_{a}\right)$ and $U\left(J^{m}\right)=U\left(\left(J^{m}\right)_{a}\right)$ by the preceding paragraph, so it follows from the hypothesis that $U(I)=U\left(I^{n}\right)=$ $U\left(\left(I^{n}\right)_{a}\right)=U\left(\left(J^{m}\right)_{a}\right)=U\left(J^{m}\right)=U(J)$.

For (2.5.7) let $P \in \hat{A}^{*}(I) \cup E(I)$. Then $P_{P} \in \hat{A}^{*}\left(I_{P}\right) \cup E\left(I_{P}\right)$, by (2.2.4), and if $P_{P} \in U\left(I_{P}\right)$, then $P \in U(I)$, by (2.5.1), so it may be assumed that $R$ is local with maximal ideal $P$. Then (2.2.6) and (2.5.3) show that $\hat{A}^{*}(I)=$ $\left\{P^{*} \cap R ; P^{*} \in \hat{A}^{*}\left(I R^{*}\right)\right\}, E(I)=\left\{P^{*} \cap R ; P^{*} \in E\left(I R^{*}\right)\right\}$, and $U(I)=\left\{P^{*} \cap R\right.$; $\left.P^{*} \in U\left(I R^{*}\right)\right\}$. Therefore it may be assumed that $R$ is complete. By (2.2.5), if $P \in E(I)$ (resp., $\hat{A}^{*}(I)$ ), then there exists $z \in$ Ass $R$ (resp., a minimal $z \in$ Ass $R$ ) such that $z \subseteq P$ and $P / z \in E\left((I+z) / z\right.$ ) (resp., $\hat{A}^{*}((I+z) / z)$ ). And if $P / z \in U((I+z) / z)$, then $P \in U(I)$, by (2.5.2), so it may be assumed that $R$ is a complete local domain. Let $\mathscr{R}=\mathscr{R}(R, I)$, so $\mathscr{R}$ is locally unmixed, by [9, Corollary, p. 61]. Then $\hat{A}^{*}(I)=\left\{p \cap R ; p \in \hat{A}^{*}(u \mathscr{R})\right\}$, by [17, (2.7)], and $\hat{A}^{*}(u \mathscr{R})=E(u \mathscr{R})$, by (2.4), so $\hat{A}^{*}(I)=U(I)$. Also, if $P \in E(I)$, then since $P$ is the maximal ideal in a complete local domain it follows from (2.1.1) that $I$ is $P$-primary. Therefore $E(I) \subseteq\{p \cap R ; p$ is a minimal prime divisor of $u \mathscr{R}\}$, since $u \mathscr{R} \cap R=I$, so $E(I) \subseteq U(I)$, by (2.4).

For (2.5.8), it was shown in (2.5.7) that $E(I) \subseteq U(I)$, so it suffices to show the other containment. For this, let $P \in U(I)$. Then by (2.5.1) $P_{P} \in$ $U\left(I_{P}\right)$, and if $P_{P} \in E\left(I_{P}\right)$, then $P \in E(I)$, by (2.2.4). Also $I_{P}$ is generated by an essential sequence, by (2.2.10), so it may be assumed that $R$ is local with maximal ideal $P$. Now $P R^{*} \in U\left(I R^{*}\right)$, by (2.5.3), and if $P R^{*} \in E\left(I R^{*}\right)$, then $P \in E(I)$, by (2.2.6). Also $I R^{*}$ is generated by an essential sequence, by (2.2.12), so it may be assumed that $R$ is a complete local ring. Now there exists $z \in$ Ass $R$ such that $P / z \in U((I+z) / z)$, by (2.5.2), and if $P / z \in$ $E((I+z) / z)$, then $P \in E(I)$, by (2.2.5). Also, $(I+z) / z$ is generated by an essential sequence, by (2.2.11), so it may be assumed that $R$ is a complete local domain. Therefore let $\mathscr{R}=\mathscr{R}(R, I)$, so $\mathscr{R}$ is locally unmixed, by [9, Corollary, p. 61]. Now $I$ is generated by an essential sequence, say $b_{1}, \cdots, b_{d}$, so height $\left(b_{1}, \cdots, b_{i}\right) R=i$ for $i=1, \cdots, d$, by (2.2.9) (since 
$b_{1}, \cdots, b_{i}$ are an essential sequence for $\left.i=1, \cdots, d\right)$. Therefore $E(I)=$ $U(I)$, by (2.4), so $P \in E(I)$.

q.e.d.

(2.5.8) together with the internal characterization of $U(I)$ mentioned just before (2.5) give an affirmative answer to a question that arose in studying essential prime divisors, namely: If $I$ is generated by an essential sequence in $R$, then is $E(I)=\cap\left\{A^{*}(J) ; I^{n} \subseteq J \subseteq\left(I^{n}\right)_{a}\right.$ for some $n \geqslant 1\}$. However, we show in (7.4) that, for general ideals $I$ in Noetherian rings, $E(I)$ may be a proper subset of this intersection.

(2.5.7) shows that $U(I)$ includes the asymptotic and essential prime divisors of $I$, and (2.5.1)-(2.5.5) show that $u$-essential prime divisors have the same nice properties these other prime divisors have in regard to passing to certain related rings. This is important, since it will be shown in (7.4) that $U(I)$ may properly contain $\hat{A}^{*}(I) \cup E(I)$.

(2.6) is a corollary of (2.5.7).

(2.6) CoRollary. If $b_{1}, \cdots, b_{d}$ are a u-essential sequence over an ideal $I$ in a Noetherian ring $R$, then $b_{1}, \cdots, b_{d}$ are an asymptotic sequence over $I$ and an essential sequence over $I$.

Proof. This is clear by (2.5.7) and (2.1.2). q.e.d.

(2.7) Remark. Let $I$ be an ideal in a Noetherian ring $R$, let $M$ be a maximal ideal in $R$ containing $I$, and let $N=(M, f) R[X]$ be a maximal ideal in $R[X]$. It may be assumed that $f$ is a monic polynomial. Then $N \in U((I, f) R[X])$ if and only if $M \in U(I)$.

Proof. Since $R[f] \cong R[X]$, it follows from (2.5.5) that $M \in U(I)$ if and only if $(M, f) R[f] \in U((I, f) R[f])$. Now $R[X]$ is integral over $R[f]$, since $f$ is monic, and $z \in$ Ass $R[X]$ implies $z \cap R[f] \in$ Ass $R[f]$. Also, $N$ is the only prime ideal in $R[X]$ that lies over $(M, f) R[f]$. Therefore $(M, f) R[f] \in$ $U((I, f) R[f])$ if and only if $N \in U((I, f) R[X])$, by (2.5.4). q.e.d.

The following lemma and its corollaries give some new information on essential prime divisors. These results will be especially useful in Section 7 where some examples are given.

(2.8) Lemma. Let $(R, M)$ be a local ring and let $I$ be an ideal in $R$. If all primary components of zero in $R^{*}$ are contained in $I R^{*}$, then $M \in$ $E(I)$ if and only if $I$ is M-primary.

Proof. If $I$ is $M$-primary, then $M \in E(I)$, by (2.2.2). 
Conversely, assume that $M \in E(I)$, let $P \in$ Ass $R / I$, and let $P^{*}$ be a minimal prime divisor of $P R^{*}$. Then $\cup\left(\right.$ Ass $\left.R^{*}\right) \subseteq P^{*}$, by hypothesis, so $I R^{*}+z \subseteq P^{*}$ for all $z \in$ Ass $R^{*}$. But $M \in E(I)$ implies that $I R^{*}+z$ is $M R^{*}$-primary for some $z \in \operatorname{Ass} R^{*}$, so $P^{*}=M R^{*}$, hence $P=M$. q.e.d.

(2.9) Corollary. If $(R, M)$ is a local ring such that Ass $R^{*}$ has exactly one element and if $I$ is an ideal in $R$, then $M \in E(I)$ if and only if $I$ is M-primary.

Proof. This is clear by (2.8). q.e.d.

(2.10) Conollary. Let $(R, M)$ be a local ring such that Ass $\left(R_{P}\right)^{*}$ has exactly one element for all $P \in \operatorname{Spec} R$. Then $E(I)=\{P \in \operatorname{Spec} R ; P$ is a minimal prime divisor of $I\}$ for all ideals $I$ in $R$.

Proof. This follows readily from (2.2.2) and (2.9), since $P \in E(I)$ implies that $P_{P} \in E\left(I_{P}\right)$.

q.e.d.

This section will be closed with the following proposition and its corollary. The proposition is a slight strengthening of [3, Corollary 2.8] and our proof below is detailed, as opposed to the sketch offered in [3]. It shows that $U(I)=\hat{A}^{*}(I)$ in a large class of Noetherian rings.

(2.11) Proposition. Let $I$ be an ideal in a Noetherian ring $R$ and assume that $\left(R_{M}\right)^{*}$ has no imbedded prime divisors of zero for all maximal ideals $M$ in $R$ that contain $I$. Then $U(I)=\hat{A}^{*}(I) \supseteq E(I)$.

Proof. It was shown in (2.5.7) that $\hat{A}^{*}(I) \cup E(I) \subseteq U(I)$, so it suffices to show that $U(I) \subseteq \hat{A}^{*}(I)$. For this let $P \in U(I)$ and let $M$ be a maximal ideal in $R$ that contains $P$. Then $P_{M} \in U\left(I_{M}\right)$, by (2.5.1), and if $P_{M} \in$ $\hat{A}^{*}\left(I_{M}\right)$, then $P \in \hat{A}^{*}(I)$, by (2.2.4), so it may be assumed that $R$ is local with maximal ideal $M$. Then if $P^{*}$ is minimal prime divisor of $P R^{*}$, then $P^{*} \in U\left(I R^{*}\right)$, by (2.5.3), and if $P^{*} \in \hat{A}^{*}\left(I R^{*}\right)$, then $P \in \hat{A}^{*}(I)$, by (2.2.6), so it may be assumed that $R$ is complete. Then there exists $z \in$ Ass $R$ such that $z \subseteq P$ and $P / z \in U((I+z) / z)$, by (2.5.2), and if $\left.P / z \in \hat{A}^{*}(I+z) / z\right)$, then $P \in \hat{A}^{*}(I)$, by (2.2.5) (since $z$ is minimal, by hypothesis), so it may be assumed that $R$ is a complete local domain. Then, by hypothesis, there exists $p \in E(u \mathscr{R})$ such that $p \cap R=P$, where $\mathscr{R}=\mathscr{R}(R, I)$. Now $\mathscr{R}$ is locally unmixed, by [9, Corollary, p. 61] (since $R$ is unmixed), so $p \in \hat{A}^{*}(u \mathscr{R})$, by (2.4). Therefore $P=p \cap R \in \hat{A}^{*}(I)$, by [17, (2.7)]. q.e.d.

(2.12) Corollary. If $I$ is an ideal in a Noetherian ring $R$ such that 
$I$ is generated by an essential sequence in $R$ and $\left(R_{M}\right)^{*}$ has no imbedded prime divisors of zero for all maximal ideals $M$ in $R$ containing $I$, then $\hat{A}^{*}(I)=E(I)=U(I)$.

Proof. This is clear by (2.5.8) and (2.11). q.e.d.

Some additional results concerning $u$-essential prime divisors will be proved in Section 5. However, their proofs require several new results, so to keep things pretty much in order of their proofs it was decided to delay giving these $u$-essential prime divisor results till the needed results have been proved.

\section{§3. U-essential sequences over an ideal}

In this section we prove several results that show that $u$-essential sequences over an ideal $I$ in a Noetherian ring $R$ behave nicely when passing to certain related rings. Then it is shown in (3.10) that $b_{1}, \cdots, b_{d}$ are a $u$-essential sequence in $R$ if and only if they are an essential sequence in $R$.

We begin with (3.1) which is essentially a corollary of (2.11). It shows that $u$-essential sequences over $I$ and asymptotic sequences over $I$ are the same in a large class of Noetherian rings.

(3.1) Theorem. Let $R$ be a Noetherian ring such that Ass $\left(R_{M}\right) *$ has no imbedded elements for all maximal ideals $M$ in $R$. Then the following hold for all ideals $I$ in $R$.

(3.1.1) $U(I)=\hat{A}^{*}(I) \supseteq E(I)$.

(3.1.2) Elements $b_{1}, \cdots, b_{d}$ in $R$ are a u-essential sequence over $I$ if and only if they are an asymptotic sequence over $I$, and this implies $b_{1}, \cdots, b_{d}$ are an essential sequence over $I$.

(3.1.3) If $R$ is local, then uecogd $(I)=\operatorname{acogd}(I) \leqslant \operatorname{ecogd}(I)$.

Proof. (3.1.1) is clear by (2.11), (3.12) follows immediately from (3.1.1), and (3.1.3) follows directly from (3.12) and (2.2.14) once it is shown that uecogd $(I)$ is well defined. This is done in (4.1). q.e.d.

In (3.3)-(3.8) we show that $u$-essential sequences over $I$ behave nicely when passing to certain related rings. The following lemma will be useful in proving these results.

(3.2) Lemma. Let $I$ be an ideal in a Noetherian ring $R$, let $b_{1}, \cdots, b_{d}$ 
be nonunits in $R$, and let $B=\left(b_{1}, \cdots, b_{d}\right) R$. Then $b_{1}, \cdots, b_{d}$ are a maximal u-essential sequence over $I$ if and only if they are a u-essential sequence over $I$ and, for each maximal ideal $M$ in $R$ containing $I+B$ it holds that $M \in U(I+B)$.

Proof. This follows readily from the definition, (2.1.2).

q.e.d.

(3.3) is concerned with $u$-essential sequences over $I$ and over $I_{S}$.

(3.3) Theorem. Let $I$ be an ideal in a Noetherian ring $R$ and let $b_{1}, \cdots, b_{d}$ be nonunits in $R$. Then the following hold:

(3.3.1) If $b_{1}, \cdots, b_{a}$ are a u-essential sequence over $I$ and $S$ is a multiplicatively closed set in $R$ such that $\left(I, b_{1}, \cdots, b_{d}\right) R_{s} \neq R_{s}$, then the images of $b_{1}, \cdots, b_{d}$ in $R_{S}$ are a u-essential sequence over $I_{s}$. The converse holds if $P_{S} \neq R_{s}$ for all $P \in \cup\left\{U\left(\left(I, b_{1}, \cdots, b_{i}\right) R\right) ; i=0,1, \cdots, d-1\right\}$.

(3.3.2) If $b_{1}, \cdots, b_{d}$ are a maximal u-essential sequence over $I$, then for each maximal ideal $M$ in $R$ that contains $\left(I, b_{1}, \cdots, b_{d}\right) R$ it holds that the images in $R_{M}$ of $b_{1}, \cdots, b_{d}$ are a maximal u-essential sequence over $I_{M}$. The converse holds if the $b_{i}$ are all in the Jacobson radical of $R$.

Proof. (3.3.1) follows immediately from (2.5.1), and the first statement in (3.3.2) follows from (2.5.1) and (3.2). For the last statement in (3.3.2) it will first be shown that $b_{1}, \cdots, b_{d}$ are a $u$-essential sequence over $I$. For this, suppose they are not, so there exists $i(0 \leqslant i<d)$ such that $b_{i+1} \in P \in U\left(\left(I, b_{1}, \cdots, b_{i}\right) R\right)$. Let $M$ be a maximal ideal in $R$ containing $P$. Then the Jacobson radical hypothesis implies that $\left(I, b_{1}, \cdots, b_{d}\right) R \subseteq M$, so the supposition and (2.5.1) imply that the image of $b_{i+1}$ is in $P_{S} \in$ $U\left(\left(I, b_{1}, \cdots, b_{i}\right) R_{s}\right)$, where $S=R-M$. But this implies that the images of $b_{1}, \cdots, b_{d}$ in $R_{M}$ are not a $u$-essential sequence over $I_{M}$, in contradiction to the hypothesis. Therefore $b_{1}, \cdots, b_{d}$ are a $u$-essential sequence over $I$. Finally, if $M$ is a maximal ideal in $R$ containing $\left(I, b_{1}, \cdots, b_{d}\right) R$ and $S=R-M$, then the hypothesis and (2.5.1) imply that $M \in$ $U\left(\left(I, b_{1}, \cdots, b_{d}\right) R\right)$, so $b_{1}, \cdots, b_{d}$ are a maximal $u$-essential sequence over $I$, by (3.2). $\quad$ q.e.d.

(3.4) is concerned with $u$-essential sequences over $I$ and over $(I+z) / z$ with $z \in$ Ass $R$.

(3.4) Theorem. Let $I$ be an ideal in a Noetherian ring $R$ and let $b_{1}, \cdots, b_{d}$ be nonunits in $R$. Then the following hold: 
(3.4.1) $b_{1}, \cdots, b_{a}$ are a u-essential sequence over $I$ if and only if their images in $R / z$ are a u-essential sequence over $(I+z) / z$ for all $z \in$ Ass $R$.

(3.4.2) $b_{1}, \cdots, b_{d}$ are a maximal $u$-essential sequence over $I$ if and only if their images in $R / z$ are a u-essential sequence over $(I+z) / z$ for all $z \epsilon$ Ass $R$ and for all maximal ideals $M$ in $R$ containing $\left(I, b_{1}, \cdots, b_{d}\right) R$ there exists $z \in$ Ass $R$ such that $z \subseteq M$ and $M / z \in U\left(\left(\left(I, b_{1}, \cdots, b_{d}\right) R+z\right) / z\right)$.

Proof. (3.4.1) follows readily from (2.5.2), and (3.4.2) follows from (2.5.2) and (3.2).

q.e.d.

(3.5) is concerned with $u$-essential sequences over $I$ and over $I A$ with $A$ a faithfully flat Noetherian $R$-algebra.

(3.5) Theorem. Let $R \subseteq A$ be Noetherian rings such that $A$ is a faithfully flat $R$-module, let $I$ be an ideal in $R$, and let $b_{1}, \cdots, b_{d}$ be nonunits in $R$. Then the following hold:

(3.5.1) $b_{1}, \cdots, b_{d}$ are a u-essential sequence over $I$ if and only if they are a u-essential sequence over IA.

(3.5.2) If $R \subseteq A$ satisfy the Theorem of Transition, then $b_{1}, \cdots, b_{d}$ are a maximal u-essential sequence over $I$ if and only if they are a maximal u-essential sequence over IA.

Proof. (3.5.1) follows readily from (2.5.3), and (3.5.2) follows from (2.5.3) and (3.2).

(3.6) is concerned with $u$-essential sequences over $I$ and over $I B$ with $B$ a finite integral extension ring.

(3.6) THEOREM. Let $B$ be a finite integral extension ring of a Noetherian ring $R$, let $I$ be an ideal in $R$, and let $b_{1}, \cdots, b_{d}$ be nonunits in $R$. Then the following hold:

(3.6.1) If $b_{1}, \cdots, b_{d}$ are a u-essential sequence over $I B$, then they are a u-essential sequence over $I$.

(3.6.2) If $z \in$ Ass $B$ implies $z \cap R \in$ Ass $R$, then $b_{1}, \cdots, b_{d}$ are $a u$ essential sequence over $I$ if and only if they are a u-essential sequence over $I B$.

(3.6.3) If $z \in$ Ass $B$ implies $z \cap R \in$ Ass $R$, then $b_{1}, \cdots, b_{d}$ are a maximal u-essential sequence over $I$ if and only if they are a u-essential sequence over IB and for each maximal ideal $M$ in $R$ containing $\left(I, b_{1}, \cdots, b_{d}\right) R$ there exists a maximal ideal $N$ in $B$ such that $N \cap R=M$ and $N \in$ $U\left(\left(I, b_{1}, \cdots, b_{d}\right) B\right)$. 
Proof. (3.6.1) and (3.6.2) follow readily from (2.5.4), and (3.6.3) follows from (2.5.4) and (3.2).

q.e.d.

(3.7) is concerned with $u$-essential sequences over $I$ and over $\operatorname{IR}[X]$.

(3.7) Theorem. Let $I$ be an ideal in a Noetherian ring $R$ and let $b_{1}, \cdots, b_{d}$ be nonunits in $R$. Then the following hold:

(3.7.1) The following are equivalent:

(a) $b_{1}, \cdots, b_{d}$ are a u-essential sequence over $I$.

(b) $b_{1}, \cdots, b_{i}, X, b_{i+1}, \cdots, b_{d}$ are a u-essential sequence over $I R[X]$ for some $i=0,1, \cdots, d$.

(c) (b) holds for every $i=0,1, \cdots, d$.

(3.7.2) The following are equivalent:

(a) $b_{1}, \cdots, b_{a}$ are a maximal u-essential sequence over $I$.

(b) $b_{1}, \cdots, b_{i}, X, b_{i+1}, \cdots, b_{d}$ are a maximal u-essential sequence over $I R[X]$ for some $i=0,1, \cdots, d$.

(c) (b) holds for every $i=0,1, \cdots, d$.

Proof. (3.7.1) For $j=0,1, \cdots i, U\left(\left(I, b_{1}, \cdots, b_{j}\right) R[X]\right)=\{P R[X] ; P \in$ $\left.U\left(\left(I, b_{1}, \cdots, b_{j}\right) R\right)\right\}$, by (2.5.3) (and since, for an ideal $J$ in $R$, the prime divisors of $J R[X]$ are the $P R[X]$ with $P$ a prime divisor of $J)$. Also, it is clear that $X$ is not in any prime divisor of $\left(I, b_{1}, \cdots, b_{i}\right) R[X]$, and, for $k=0,1, \cdots, d-i, U\left(\left(I, b_{1}, \cdots, b_{i}, X, b_{i+1}, \cdots, b_{i+k}\right) R[X]\right)=\{(P, X) R[X] ;$ $\left.P \in U\left(\left(I, b_{1} \cdot \cdots, b_{i+k}\right) R\right)\right\}$, by (2.5.5). Therefore it follows that (3.7.1) (a)-(c) are equivalent.

(3.7.2) follows immediately from (3.7.1) and (3.2), since the maximal ideals in $R[X]$ containing $(I, X) R[X]$ are the ideals $(M, X) R[X]$ with $M$ a maximal ideal in $R$ containing $I$.

q.e.d.

(3.8) is concerned with $u$-essential sequences over projectively equivalent ideals. Remark (3.9) below is required for its proof.

(3.8) Theorem. Let $I$ and $J$ be ideals in a Noetherian ring $R$ such that $\left(I^{n}\right)_{a}=\left(J^{m}\right)_{a}$ for some $n \geqslant 1$ and $m \geqslant 1$ and let $b_{1}, \cdots, b_{d}$ be nonunits in $R$. Then the following hold:

(3.8.1) $b_{1}, \cdots, b_{d}$ are a u-essential sequence over $I$ if and only if they are a u-essential sequence over $J$.

(3.8.2) $b_{1}, \cdots, b_{d}$ are a maximal u-essential sequence over $I$ if and only if they are a maximal u-essential sequence over $J$. 
Proof. (3.8.1) Note first that for each $i=0,1, \cdots, d$ we have $\left(\left(I, b_{1}\right.\right.$, $\left.\left.\cdots, b_{i}\right)^{n} R\right)_{a}=\left(\left(\left(I^{n}\right)_{a}, b_{1}^{n}, b_{2}^{n}, \cdots, b_{i}^{n}\right) R\right)_{a}$, so $H=\left(I, b_{1}, \cdots, b_{i}\right) R$ and $K=$ $\left(\left(I^{n}\right)_{a}, b_{1}^{n}, \cdots, b_{i}^{n}\right) R$ are projectively equivalent. Therefore $U(H)=U(K)$, by (2.5.6), so $b_{i+1} \notin \cup U(H)$ if and only if $b_{i+1} \notin \cup U(K)$ if and only if $b_{i+1}^{n} \notin \cup U(K)$. Therefore it follows that $b_{1}, \cdots, b_{d}$ are a $u$-essential sequence over $I$ if and only if $b_{1}^{n}, \cdots, b_{d}^{n}$ are a $u$-essential sequence over $\left(I^{n}\right)_{a}$. And, similarly, $b_{1}, \cdots, b_{d}$ are a $u$-essential sequence over $J$ if and only if $b_{1}^{m}, \cdots, b_{d}^{m}$ are $a u$-essential sequence over $\left(J^{m}\right)_{a}$. Therefore $b_{1}, \cdots, b_{d}$ are $a u$-essential sequence over $I$ if and only if $b_{1}^{n}, \cdots, b_{d}^{n}$ are a $u$-essential sequence over $\left(I^{n}\right)_{a}$ if and only if $u, b_{1}^{n}, \cdots, b_{d}^{n}$ are an essential sequence in $\mathscr{R}=\mathscr{R}\left(R,\left(I^{n}\right)_{a}\right)$, by (3.9), and this holds if and only if $u, b_{1}^{m}, \cdots, b_{d}^{m}$ are an essential sequence in $\mathscr{R}$, by (2.2.8). Now $\mathscr{R}=\mathscr{R}\left(R,\left(J^{m}\right)_{a}\right)$, by hypothesis, so this holds if and only if $b_{1}^{m}, \ldots, b_{d}^{m}$ are a $u$-essential sequence over $\left(J^{m}\right)_{a}$, by (3.9), if and only if $b_{1}, \cdots, b_{d}$ are a $u$-essential sequence over $J$, as noted above. Therefore (3.8.1) holds.

(3.8.2) follows immediately from (3.8.1) and (3.2).

(3.9) Remark. [21]. If $I$ is an ideal in a Noetherian ring $R$ and $b_{1}, \cdots, b_{d}$ are nonunits in $R$, then $b_{1}, \cdots, b_{d}$ are a $u$-essential sequence over $I$ if and only if $u, b_{1}, \cdots, b_{d}$ are an essential sequence in $\mathscr{R}(R, I)$.

In (3.10) we show that $u$-essential sequences and essential sequences are the same (cf. [3] concluding Remark 3).

(3.10) Proposition. Let $b_{1}, \cdots, b_{d}$ be nonunits in a Noetherian ring $R$. Then $b_{1}, \cdots, b_{d}$ are a u-essential sequence in $R$ if and only if they are an essential sequence in $R$.

Proof. It follows immediately from (2.6) (applied to $I=(0)$ ) that a $u$-essential sequence in $R$ is an essential sequence.

The converse is immediate from (2.5.8) and (2.1.2). q.e.d.

Because of (3.10) we will not henceforth talk about $u$-essential sequences in $R$. However, it is shown in (7.1) and its preceding comment that $u$-essential sequences over $I$ are different from essential sequences over $I$, so it is necessary to use this terminology.

This section will be closed with the following remark which gives some additional basic properties of $u$-essential sequences over $I$.

(3.11) Remark. Let $I$ be an ideal in a Noetherian ring $R$ and let $b_{1}, \cdots, b_{d}$ be nonunits in $R$. Then the following hold: 
(3.11.1) The following statements are equivalent:

(a) $b_{1}, \cdots, b_{d}$ are a $u$-essential sequence over $I$; (b) $b_{1}^{n_{1}}, \cdots, b_{d}^{n_{d}}$ are a $u$-essential sequence over $I$ for some positive integers $n_{i}$; (c) (b) holds for all positive integers $n_{i}$.

(3.11.2) The following statements are equivalent:

(a) $b_{1}, \cdots, b_{d}$ are a $u$-essential sequence over $I$; (b) There exists an $i(0 \leqslant i<d)$ such that $b_{1}, \cdots, b_{i}$ are a $u$-essential sequence over $I$ and $b_{i+1}, \cdots, b_{d}$ are a $u$-essential sequence over $\left(I, b_{1}, \cdots, b_{i}\right) R$; (c) (b) holds for all $i(i=0,1, \cdots, d-1)$.

Proof. (3.11.1) $b_{1}, \cdots, b_{d}$ are a $u$-essential sequence over $I$ if and only if $u, b_{1}, \cdots, b_{d}$ are an essential sequence in $\mathscr{R}=\mathscr{R}(R, I)$, by (3.9), if and only if $u, b_{1}^{n_{1}}, \cdots, b_{d}^{n_{d}}$ are an essential sequence in $\mathscr{R}$, by [19, (2.11.1)], if and only if $b_{1}^{n_{1}}, \cdots, b_{d}^{n_{d}}$ are a $u$-essential sequence over $I$, by (3.9), so (3.11.1) holds.

(3.11.2) is clear by the definition, (2.1.2). q.e.d.

\section{$\S 4$. On the $U$-essential cograde of an ideal}

In this section we show that uecogd $(I)$ is unambiguously defined for ideals $I$ in a local ring $R$, we give one characterization of this cograde, and we then show that it behaves nicely when passing to certain ideals related to $I$.

We begin by showing uecogd $(I)$ is well defined. In (4.1), $\ell(J)$ denotes the analytic spread of the ideal $J$.

(4.1) Theorem. If $I$ is an ideal in a local ring $R$, then any two maximal u-essential sequences over I have the same length, so uecogd $(I)$ is unambiguously defined. Moreover, uecogd $(I)=\min \left\{\operatorname{depth} z-\ell\left(\left(I R^{*}+z\right) / z\right)\right.$; $\left.z \in \operatorname{Ass} R^{*}\right\}$.

Proof. Let $b_{1}, \cdots, b_{d}$ be a maximal $u$-essential sequence over $I$. Then by (3.5.2) and (3.4.2) their images in $R^{*} / z$ are $a u$-essential sequence over $\left(I R^{*}+z\right) / z$ for all $z \in$ Ass $R^{*}$ and for some such $z$ their images are a maximal $u$-essential sequence over $\left(I R^{*}+z\right) / z$. Therefore their images are an asymptotic sequence over $\left(I R^{*}+z\right) / z$ for all $z \in$ Ass $R^{*}$ and for some such $z$ their images are maximal asymptotic sequence over $\left(I R^{*}+z\right) / z$, by (3.1.2). Now, for each $z \in$ Ass $R^{*}$ it holds that acogd $\left(\left(I R^{*}+z\right) / z=\right.$ depth $z-\ell\left(\left(I R^{*}+z\right) / z\right)$, by (2.2.14), so it follows that $d \leqslant \operatorname{depth} z-\ell\left(\left(I R^{*}+\right.\right.$ $z) / z$ ) for all $z \in$ Ass $R^{*}$ and equality holds for some such $z$, so $d=$ 
$\min \left\{\operatorname{depth} z-\ell\left(\left(I R^{*}+z\right) / z\right) ; z \in \operatorname{Ass} R^{*}\right\}$.

q.e.d.

(4.2) Corollary. Let $I$ be an ideal in a local ring $(R, M)$ and let $\mathscr{S}=\mathscr{R}\left(R^{*}, I R^{*}\right)$. Then uecogd $(I)=\min \left\{\right.$ height $\left(M R^{*} / z, u\right) \mathscr{S} / z^{*} ; z \in$ Ass $R^{*}$ and $\left.z^{*}=z R^{*}[t, u] \cap \mathscr{S}\right\}-1$.

Proof. By definition, $\ell\left(\left(I R^{*}+z\right) / z\right)=\operatorname{depth}\left(M R^{*} \mid z, u\right) \mathscr{T}$, where $\mathscr{T}=$ $\mathscr{R}\left(R^{*} / z,\left(I R^{*}+z\right) / z\right)$, so $\mathscr{T} \cong \mathscr{S} / z^{*}$, by [22, Lemma 1.1]. Also, each ring $\mathscr{S} / z^{*}$ is locally quasi-unmixed, so $\left(\mathscr{S} \mid z^{*}\right)_{\mathscr{K} / z^{*}}$ satisfies the first chain condition for prime ideals where $\mathscr{M}$ is the maximal homogeneous ideal in

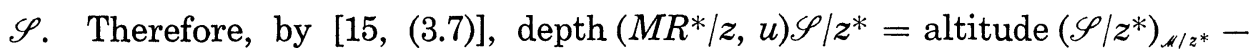
height $\left(M R^{*} / z, u\right) \mathscr{S} / z^{*}$. Further, altitude $\left(\mathscr{S} / z^{*}\right)_{\alpha^{*} z^{*}}=\operatorname{depth} z+1$, by [15, (2.2.4)]. Therefore by (4.1) it follows that uecogd $(I)=\min \{\operatorname{depth} z-$ $\left.\ell\left(\left(I R^{*}+z\right) / z\right) ; z \in \operatorname{Ass} R^{*}\right\}=\min \left\{\operatorname{depth} z-(\operatorname{depth} z+1)+\operatorname{height}\left(M R^{*} / z, u\right)\right.$ $\mathscr{S} \mid z^{*} ; z \in$ Ass $\left.R^{*}\right\}=\min \left\{\right.$ height $\left(M R^{*} / z, u\right) \mathscr{S} / z^{*} ; z \in$ Ass $\left.R^{*}\right\}-1$. q.e.d.

(4.3) shows that uecogd $(I)$ behaves nicely when passing to certain related rings and ideals.

(4.3) Theorem. Let $I$ be an ideal in a local ring $(R, M)$. Then the following hold:

(4.3.1) uecogd $(I)=\min \{\operatorname{uecogd}((I+z) / z) ; z \in$ Ass $R\}$.

(4.3.2) If $A$ is a faithfully flat Noetherian extension ring of $R$, then uecogd $(I) \leqslant \operatorname{uecogd}\left(I A_{N}\right)$ for all prime ideals $N$ in $A$ lying over $M$ and equality holds if height $N=$ height $M$.

(4.3.3) If $B$ is a finite integral extension ring of $R$ such that $z \in \operatorname{Ass} B$ implies $z \cap R \in$ Ass $R$, then uecogd $(I) \leqslant$ uecogd $\left(I B_{N}\right)$ for all maximal ideals $N$ in $B$ and equality holds for some such $N$.

(4.3.4) If $J$ is projectively equivalent to $I$ then uecogd $(J)=\operatorname{uecogd}(I)$.

Proof. These statements follow immediately from, respectively, (3.4.2), (3.5) and (3.3.1), (3.6) and (3.3.1), and (3.8.2).

q.e.d.

\section{§5. Preliminaries for bounds on uecogd (I)}

In this section we prove three theorems, and several corollaries of one of them, that are used in Section 6 to establish certain bounds on uecogd $(I)$.

We begin with the $u$-essential analogue of $[6,(3.2)]$, which gives a nice containment relation between ideals in $\hat{A}^{*}(I)$ and in $\hat{A}\left(\left(I, b_{1}, \cdots, b_{d}\right) R\right)$, where $b_{1}, \cdots, b_{d}$ are an asymptotic sequence over $I$. 
(5.1) Theorem. Let $I$ be an ideal in a Noetherian ring $R$, let $P \in U(I)$, let $b_{1}, \cdots, b_{a}$ be a u-essential sequence over $I$, and let $N$ be a minimal prime divisor of $\left(P, b_{1}, \cdots, b_{d}\right) R$. Then $N \in U\left(\left(I, b_{1}, \cdots, b_{d}\right) R\right)$.

Proof. By (2.5.1) $P_{N} \in U\left(I_{N}\right)$ and if $N_{N} \in U\left(\left(I, b_{1}, \cdots, b_{d}\right) R_{N}\right)$, then $N \in U\left(\left(I, b_{1}, \cdots, b_{d}\right) R\right)$. Also, the images of $b_{1}, \cdots, b_{d}$ in $R_{N}$ are a $u$ essential sequence over $I$, by (3.3.1), so it may be assumed that $R$ is local with maximal ideal $N$. Then by (2.5.3) if $P^{*}$ is a minimal prime divisor of $P R^{*}$, then $P^{*} \in U\left(I R^{*}\right)$ and if $N R^{*} \in U\left(\left(I, b_{1}, \cdots, b_{d}\right) R^{*}\right)$, then $N \in$ $U\left(\left(I, b_{1}, \cdots, b_{d}\right) R\right)$. Also, $b_{1}, \cdots, b_{d}$ are a $u$-essential sequence over $I R^{*}$, by (3.5.1), so it may be assumed that $R$ is complete. By (2.5.2) there exists $z \in$ Ass $R$ such that $z \subseteq P$ and $P / z \in U((I+z) / z)$ and if $N / z \in U\left(\left(\left(I, b_{1}\right.\right.\right.$, $\left.\left.\left.\cdots, b_{d}\right) R+z\right) / z\right)$, then $N \in U\left(\left(I, b_{1}, \cdots, b_{d}\right) R\right)$. Also, the images of $b_{1}, \cdots, b_{d}$ are a $u$-essential sequence over $(I+z) / z$, by (3.4.1), so it may be assumed that $R$ is a complete local domain.

Let $\mathscr{R}=\mathscr{R}(R, I)$ and let $p \in E(u \mathscr{R})$ such that $p \cap R=P$. Then height $p=1$, by (2.4), and $u, b_{1}, \cdots, b_{d}$ are an essential sequence in $\mathscr{R}$, by (3.9). Therefore, if $q$ is a minimal prime divisor of $\left(p, b_{1}, \cdots, b_{d}\right) \mathscr{R}$, then height $q=d+1$, by (2.2.9) and since $\mathscr{R}$ is catenary. Also, $b_{i} / u=t b_{i}$, so $q^{\prime}=q \mathscr{R}\left[t b_{1}, \cdots, t b_{d}\right]$ is a height one prime divisor of $u \mathscr{R}\left[t b_{1}, \cdots, t b_{d}\right]$ that lies over $q$, by [14, Lemma 2.7]. Therefore, since $\mathscr{R}\left[t b_{1}, \cdots, t b_{d}\right]=$ $\mathscr{R}\left(R,\left(I, b_{1}, \cdots, b_{d}\right) R\right), q^{\prime} \cap R \in U\left(\left(I, b_{1}, \cdots, b_{d}\right) R\right)$. Also, $q^{\prime} \cap R=N$, since $q^{\prime} \cap R=q \cap R \supseteq\left(P, b_{1}, \cdots b_{d},\right) R$.

q.e.d.

(5.2) Corollary. Let $I$ be an ideal in a Noetherian ring $R$ and let $b_{1}, \cdots, b_{d}$ be a u-essential sequence over $I$. Then, for $i=0,1, \cdots, d-1$, given any $P \in U\left(\left(I, b_{1}, \cdots, b_{i}\right) R\right)$ there exists $Q \in U\left(\left(I, b_{1}, \cdots, b_{i+1}\right) R\right)$ such that $P \subset Q$. Moreover, if $R$ is locally quasi-umnixed, then $Q$ can be chosen such that height $Q=$ height $P+1$.

Proof. The first statement follows immediately from (5.1), and the last statement follows from the first chain condition for prime ideals in $R_{Q}$ in the quasi-unmixed case.

q.e.d.

(5.3) is a $E(I)$ analogue of (5.2).

(5.3). Corollary. Let $b_{1}, \cdots, b_{d}$ be an essential sequence in a Noetherian ring $R$, fix $i(0 \leqslant i<d)$, let $P \in E\left(\left(b_{1}, \cdots, b_{i}\right) R\right)$, and let $N$ be $a$ minimal prime divisor of $\left(P, b_{i+1}, \cdots, b_{d}\right) R$. Then $N \in E\left(\left(b_{1}, \cdots, b_{d}\right) R\right)$.

Proof. $E\left(\left(b_{1}, \cdots, b_{j}\right) R\right)=U\left(\left(b_{1}, \cdots, b_{j}\right) R\right)$ for $j=1, \cdots, d$, by (2.5.8). 
Therefore, if $I=\left(b_{1}, \cdots, b_{i}\right) R$, then $b_{i+1}, \cdots, b_{d}$ are a $u$-essential sequence over $I$, so the conclusion follows immediately from (5.1). q.e.d.

(5.3) is definitely a weaker result than (5.1), and it is shown in (7.3) that the essential sequence over $I$ analog of (5.1) is not true.

(5.4) is the $u$-essential analogue of the following result: If $P \in \hat{A}^{*}(B)$, where $B$ is generated by an asymptotic sequence, then $P \in \hat{A}^{*}(I)$ for all ideals $I$ in $R$ such that $B \subseteq \operatorname{Rad} I \subseteq P$.

(5.4) Theorem. Let $B \subseteq P$ be ideals in a Noetherian ring $R$ such that $B$ is generated by an essential sequence in $R$ and $P \in U(B)$. Then $P \in U(I)$ for all ideals $I$ in $R$ such that $B \subseteq \operatorname{Rad} I \subseteq P$.

Proof. By (3.11.1) and the hypothesis that $B \subseteq \operatorname{Rad} I$ it may be assumed that $B \subseteq I$. Then by (2.5.1) and (2.2.10) it may be assumed that $R$ is local with maximal ideal $P$, and then by (2.5.3) and (2.2.12) it may be assumed that $R$ is a complete local ring. Finally, by (2.5.2) and (2.2.11) it may be assumed that $R$ is a complete local domain. Then $P \in U(B)$ implies $P$ is a minimal prime divisor of $B$, by (2.4), so $B$ is $P$-primary. Thus $I$ is $P$-primary, so $P \in U(I)$, by (2.3.2).

q.e.d.

(5.5) contains several remarks that will be used in the proof of (5.6) and (6.7)-(6.9).

(5.5) Remark. The following hold for an ideal $I$ in a local ring $R$ :

(5.5.1) If $b_{1}, \cdots, b_{d}$ in $R$ are a $u$-essential sequence over $I$, then $\ell\left(\left(I, b_{1}, \cdots, b_{a}\right) R\right)=\ell(I)+d$.

(5.5.2) If $b_{1}, \cdots, b_{d}$ are nonunits in $R$ whose images modulo $I^{n}$ are an $R / I^{n}$-sequence for all large $n$, then $\ell\left(\left(\left(I, b_{1}, \cdots, b_{d}\right) R\right) /\left(b_{1}, \cdots, b_{d}\right) R\right)=\ell(I)$.

(5.5.3) For all large $k$ it holds that grade $R / I^{n}=$ grade $R / I^{k}$ for all $n \geqslant k$ and if $b_{1}, \cdots, b_{d}$ are nonunits in $R$ whose images in $R / I^{k}$ are an $R / I^{k}$-sequence, then their images in $R / I^{n}$ are an $R / I^{n}$-sequence for all $n \geqslant k$.

(5.5.4) If $J$ is an ideal in $R$, then $\ell(I) \geqslant \ell((I+J) / J)$.

Proof. For (5.5.1), $b_{1}, \cdots, b_{d}$ are an asymptotic sequence over $I$, by (2.6), so the conclusion is given by [6, (3.1)].

(5.5.2) and (5.5.3) are given by [6, (7.1) and (7.3)].

For (5.5.4) let $\mathscr{R}=\mathscr{R}(R, I)$ and let $\mathscr{S}=\mathscr{R}(R / J,(I+J /) J)$, so $\mathscr{S} \cong \mathscr{R} / J^{*}$ where $J^{*}=J R[t, u] \cap \mathscr{R}$, by [22, Lemma 1.1]. Let $p$ be a minimal prime 
divisor of $(M / J, u) \mathscr{S}$ such that depth $p=\ell((I+J) / J)$ and let $P$ be the pre-image of $p$ in $\mathscr{R}$. Then $(M, u) \mathscr{R} \subseteq P$, so $\ell(I)=\operatorname{depth}(M, u) \mathscr{R} \geqslant$ $\operatorname{depth} P=\operatorname{depth} p=\ell((I+J) / J)$.

q.e.d.

(5.6) is the final result in this section. Its proof is similar to the proof of $[6,(7.2)]$, but there are enough differences that it was decided to include the details here.

(5.6) Theorem. Let $I$ be an ideal contained in the Jacobson radical of a locally unmixed Noetherian ring $R$ and assume that $b_{1}, \cdots, b_{d}$ are elements in $R$ whose images in $R / I^{n}$ are an $R / I^{n}$-sequence for all large $n$. Then $b_{1}, \cdots, b_{d}$ are a u-essential sequence over $I$.

Proof. The hypothesis on $b_{1}$ implies that $b_{1} \notin \cup A^{*}(I)=\cup\{P \in \operatorname{Spec} R$; $P$ is a prime divisor of $I^{n}$ for all large $\left.n\right\}$. Therefore, since $U(I) \subseteq A^{*}(I)$, by (2.3.3), it follows that $b_{1}$ is a $u$-essential sequence over $I$.

We now inductively assume that $b_{1}, \cdots, b_{d-1}$ are a $u$-essential sequence over $I$. Then it is shown in [21] that $b_{1}, \cdots, b_{d-1}$ are an essential sequence in $R$, so height $\left(b_{1}, \cdots, b_{d-1}\right) R=d-1$, by (2.2.9). We must show that $b_{a} \notin \cup U\left(\left(I, b_{1}, \cdots, b_{d-1}\right) R\right)$, so suppose, on the contrary, that $b_{d} \in P$ for some $P \in U\left(\left(I, b_{1}, \cdots, b_{d-1}\right) R\right)$. Then it may be assumed that $R$ is local with maximal ideal $P$, by (2.5.1). Now $U(J)=\hat{A}^{*}(J)$ for all ideals $J$ in $R$, by (3.1.1), so $P \in \hat{A}^{*}\left(\left(I, b_{1}, \cdots, b_{d-1}\right) R\right)$, and so height $P=\ell\left(\left(I, b_{1}, \cdots, b_{d-1}\right) R\right)$ $=\ell(I)+d-1$, by [5, Theorem 3] and (5.5.1). Let' denote residue class modulo $\left(b_{1}, \cdots, b_{d-1}\right) R$. Then $\ell\left(I^{\prime}\right)=\ell(I)=$ height $P-d+1$, by (5.5.2). Also, height $\left(b_{1}, \cdots, b_{d-1}\right) R=d-1$, as already noted, so by the first chain condition for prime ideals in $R$ (since $R$ is unmixed) it follows that height $P^{\prime}=$ height $P-d+1$. Therefore $\ell\left(I^{\prime}\right)=$ height $P^{\prime}$. Now every minimal prime divisor of $\left(b_{1}, \cdots, b_{d-1}\right) R$ has height $d-1$, so $R^{\prime}$ is quasi-unmixed, by [11, Corollary 2.2]. Therefore $P^{\prime} \in \hat{A}^{*}\left(I^{\prime}\right) \subseteq A^{*}\left(I^{\prime}\right)$, by [5, Theorem 3] and (2.2.1) and so $P \in\left\{Q \in \operatorname{Spec} R ; Q\right.$ is a prime divisor of $I^{n}+\left(b_{1}, \cdots, b_{d-1}\right) R$ for all large $n\}$. However, $b_{d} \in P$, and this contradicts the hypothesis on $b_{1}, \cdots, b_{d}$, so $b_{1}, \cdots, b_{d}$ are a $u$-essential sequence over $I$.

q.e.d.

\section{§6. Some bounds for uecogd $(I)$}

In this section we show that most of the results in [6] concerning $\operatorname{acogd}(I)$ have a valid uecogd $(I)$ analogue. We begin with the following remark. 
(6.1) Remark. If $R$ is a local ring, then the following hold:

(6.1.1) If $R^{*}$ has no imbedded prime divisors of zero, then uecogd $(I)=$ acogd (I) for all ideals $I$ in $R$, by (3.1.3). Therefore in this case all the results in [6] concerning acogd $(I)$ hold for uecogd $(I)$.

(6.1.2) It follows immediately from (2.6) that, in general, uecogd $(I) \leqslant$ $\operatorname{acogd}(I)$ and uecogd $(I) \leqslant \operatorname{ecogd}(I)$. We show in (7.4) that both inequalities can hold.

Even when the hypothesis in (6.1.1) is not satisfied, most of the bounds on $\operatorname{acogd}(I)$ in [6] have a valid uecogd $(I)$ analogue, as we now show. The first of these bounds is the analogue of [6, (3.5)]: acogd $(I) \leqslant$ $\min \left\{\right.$ little depth $\left.P ; P \in \hat{A}^{*}(I)\right\}$. Here, little depth $P$ is the length of a shortest maximal chain of prime ideals in $R / P$.

(6.2) THeOREM. If $I$ is an ideal in a local ring $(R, M)$, then uecogd $(I) \leqslant \min \{$ little depth $P ; P \in U(I)\}$. Therefore, if $A$ is a faithfully fiat local extension ring of $R$, then uecogd $(I) \leqslant \min \left\{\right.$ little depth $P^{*} ; P^{*} \in$ $U(I A)\}$.

Proof. The proof is essentially the same as that given to prove the asymptotic cograde case in $[6,(3.5)]$, but use (5.1) in place of [6, (3.2)].

$$
\text { q.e.d. }
$$

(6.3) Remark. Equality need not hold in (6.2) even when $R$ is a complete local domain and $I$ is a height one prime ideal such that $U(I)=\{I\}$.

Proof. [6, (3.6)] shows that equality need not hold in this case for asymptotic cograde. Therefore the conclusion follows, since $U(I)=\hat{A}^{*}(I)$ and $\operatorname{uecogd}(I)=\operatorname{acogd}(I)$ when $R$ is a complete local domain, by (3.1).

$$
\text { q.e.d. }
$$

The next bound on acogd (I) in [6] depends on the fact that if $b_{1}, \cdots, b_{g}$ are an asymptotic sequence over $I$, then their images in $R / I$ are an asymptotic sequence. The $u$-essential sequence over $I$ version of this doss not hold, as noted in [7, (7.1)]. Therefore the uecogd $(I)$ analogue of $[6$, (4.5)]: $\operatorname{acogd}(I) \leqslant \operatorname{agd}(M / I)$; does not hold. In fact, if $I$ is an ideal in $R$ such that $M \in \operatorname{Ass} R / I, \notin U(I)$, then $\operatorname{uecogd}(I) \geqslant 1>0=\operatorname{egd}(M / I)$. Therefore, since uecogd $(I)=\operatorname{acogd}(I)$ when $R^{*}$ has no imbedded prime divisors of zero, (6.4) is the best possible uecogd (I) analogue of $[6,(4.5)]$.

(6.4) Theorem. If $I$ is an ideal in a local ring $R$, then uecogd $(I) \leqslant$ $\operatorname{agd}(M / I)$. 
Proof. This follows immediately from [6, (4.5)] and (6.1.2). q.e.d.

(6.5) is the uecogd $(I)$ version of $[6,(6.1)]: \operatorname{acogd}(I) \leqslant \operatorname{agd}(M)-\operatorname{agd}(I)$.

(6.5) Theorem. Let $I$ be an ideal in a local ring $(R, M)$ and let $x_{1}, \cdots, x_{h}$ be an essential sequence in $I$. Then there exists a maximal $u$ essential sequence over $I$, say $b_{1}, \cdots, b_{d}$, such that $x_{1}, \cdots, x_{h}, b_{1}, \cdots, b_{d}$ are an essential sequence in $R$. In particular, uecogd $(I) \leqslant \operatorname{egd}(M)-\operatorname{egd}(I)$.

Proof. The proof is the same as that given to prove [6, (6.1)], but use (5.5) to show that $M \notin U(I)$ implies $M \notin U\left(\left(x_{1}, \cdots, x_{h}\right) R\right)$. q.e.d.

(6.6) is the uecogd $(I)$ analogue of $[6,(6.2)]$.

(6.6) TheOREM. The following statements are equivalent for an ideal $I$ in a local ring $(R, M)$ :

(6.6.1) uecogd $(I)=\operatorname{egd}(M)-\operatorname{egd}(I)$.

(6.6.2) There exists $z \in$ Ass $R^{*}$ such that $\ell\left(\left(I R^{*}+z\right) / z\right)=$ height $\left(I R^{*}\right.$ $+z) / z=\operatorname{egd}(I)$ and uecogd $(I)=\operatorname{depth} z-\operatorname{height}\left(I R^{*}+z\right) / z$.

(6.6.3) The equalities in (6.6.2) hold for every $z \in$ Ass $R^{*}$ such that depth $z=\operatorname{egd}(M)$.

Proof. The proof is the same as that given to prove [6, (6.2]), except one must substitute for the asymptotic-references the analogous essentialreferences.

q.e.d.

The final bounds on acogd $(I)$ in [6] are lower bounds. To prove the uecogd $(I)$ version of these we will use (5.5) and (5.6).

(6.7) is the uecogd $(I)$ version of $[6,(7.4)]:$ If $(R, M)$ is a quasi-unmixed local ring, then acogd $(I) \geqslant$ grade $M / I^{n}$ for all large $n$.

(6.7) Theorem. If $I$ is an ideal in an unmixed local ring $(R, M)$, then uecogd $(I) \geqslant \operatorname{grade}\left(M / I^{n}\right)$ for all large $n$.

Proof. This is clear by (5.6) and (5.5.3). q.e.d.

(6.8) Remark. If $I$ is an ideal in an unmixed local ring $(R, M)$, then grade $M / I^{n} \leqslant \operatorname{uecogd}(I) \leqslant \operatorname{agd}\left(M / I^{n}\right)$ for all large $n$.

Proof. It follows from $[18,(2.13)]$ that $\operatorname{agd}\left(M / I^{n}\right)=\operatorname{agd}(M / I)$ for all $n \geqslant 1$, so this follows immediately from (6.4) and (6.7). q.e.d.

The final result in this section, (6.9), is the uecogd $(I)$ analogue of $[6,(7.6)]: \operatorname{acogd}(I) \geqslant \operatorname{agd}(M)-\ell(I)$. 
(6.9) Theorem. If $I$ is an ideal in a local ring $(R, M)$, then uecogd $(I)$ $\geqslant \operatorname{egd}(M)-\ell(I)$.

Proof. By (4.1) let $z \in$ Ass $R^{*}$ such that uecogd $(I)=$ height $M R^{*} / z-$ $\ell\left(\left(I R^{*}+z\right) / z\right.$. Then height $M R^{*} / z \geqslant \operatorname{egd}(M)$, by $(2.2 .13)$, and $\ell\left(\left(I R^{*}+z\right) z\right)$ $\leqslant \ell(I)$, by (5.5.4), so the conclusion follows.

q.e.d.

\section{§. Some examples}

In this final section we give four examples that show some of the differences between essential sequences over $I$ and $u$-essential sequences over $I$.

(7.1) shows that a permutation of an essential sequence over an ideal $I$ in a local ring need not be an essential sequence over $I$, even though this holds for $u$-essential sequences over $I$, as is shown in [21].

(7.1) EXAMPLE. There exists a local domain $R$ such that $R$ has an ideal $I$ and elements $b_{1}, b_{2}$ such that $b_{1}, b_{2}$ are an essential sequence over $I$ and $b_{2}, b_{1}$ are not.

Proof. Let $R$ be a complete regular local ring of altitude three and let $a, b, c$ be a regular system of parameters in $R$. Let $p=(b-c) R$, $P=(a, c) R$, and $I=p \cap P$, so $I=p(P: p)=p P=\left(a b-a c, b c-c^{2}\right) R$. Then $E(I)=\{p, P\}$, by (2.10), and $b, a$ are an essential sequence over $I$ (since $b$ is prime to $I$ and $E((I, b) R)=E\left(\left(a c, c^{2}, b\right) R\right)=\{(b, c) R\}$, by $(2.10)$ ), but $a \in P$, so $a, b$ are not an essential sequence over $I$. Thus let $b_{1}=b$ and $b_{2}=a$.

q.e.d.

(7.2) shows that an essential sequence over an ideal $I$ in a local domain $R$ need not be an essential sequence in $R$, but a $u$-essential sequence over $I$ is an essential sequence in $R$, as is shown in [21].

In the proof of (7.2) (and also in (7.4)) we again use $\ell(I)$ to denote the analytic spread of an ideal $I$.

(7.2) ExAmple. There exists a local domain $R$ such that $R$ has an ideal $I$ and elements $b_{1}, b_{2}$ such that $b_{1}, b_{2}$ are an essential sequence over $I$ and $b_{1}, b_{2}$ are not an essential sequence in $R$.

Proof. Let $(L, M=(a, b) L)$ be a complete regular local ring of altitude two, let $A=L[t a, t b]$, where $t$ is an indeterminate, and let $R=A_{(M, t a, t b) A}$, so $R$ is a local domain of altitude three. It will first be shown that $M^{n} R$ is $M R$-primary for $n \geqslant 1$. 
For this, let $\mathscr{R}=\mathscr{R}(L, M)=L[t a, t b, u]=A[u]$, with $u=1 / t$. Then $\mathscr{R} / u \mathscr{R}=A / M A=\mathscr{F}(L, M)$, the form ring of $L$ with respect to $M$, by [22, Theorem 2.1], and $\mathscr{F}(L, M)$ is an altitude two integral domain, since $L$ is regular, so $u \mathscr{R}$ and $M A$ are height one depth two prime ideals. Also, $u^{n} \mathscr{R} \cap A=M^{n} A$ for all $n \geqslant 1$, so $M^{n} A$ is integrally closed for all $n \geqslant 1$, since $u^{n} \mathscr{R}$ is (since $u \mathscr{R}$ is prime). Therefore $N=(M, t a, t b) R$ is not a prime divisor of $M^{n} R$ for all $n \geqslant 1$, by [5, Theorem 3] (since $\ell\left(M^{n} R\right)=\ell(M R)$ and $\ell(M R) \leqslant 2$ (since $M$ is generated by two elements) and since $R$ is quasi-unmixed (since $L$ is)). Also, if $P \in \operatorname{Spec} R$ and $P \neq N$, then $R_{P}$ is regular, since $A[1 / t]=\mathscr{R}(L, M)$ is locally regular, by [23, Theorem 2], and $A[t]=L[t]$ is locally regular. Therefore, if height $P=2$, then $P$ is not a prime divisor of $M^{n} R$ for all $n \geqslant 1$ (since $M^{n} R_{P}$ is principal). Therefore $A^{*}(M R)=\{M R\}$, so $E(M R)=\{M R\}$, by $(2.2 .1)$.

Therefore it follows that $R / M R \simeq(L / M)[X, Y]_{(X, Y)}$, and $R^{*} / M R^{*} \cong$ $(R / M R)^{*}$, so $M R^{*}$ is prime Also, it was just shown that $M^{n} R$ is $M R$ primary for all $n \geq 1$, so it follows from flatness that $M^{n} R^{*}$ is $M R^{*}$-primary for all $n \geq 1$, so $A^{*}\left(M R^{*}\right)=\left\{M R^{*}\right\}$, hence $E\left(M R^{*}\right)=\left\{M R^{*}\right\}$, by (2.2.1). Thus it readily follows from (2.2.3) that every prime divisor of zero in $R^{*}$ is contained in $M R^{*}$, so this also holds for $(M, t a) R^{*}$, and so $E((M, t a) R)=$ $\{(M, t a) R\}$, by (2.8). Therefore $t a, t b$ are an essential sequence over $M R$, but $(t a, t b) R=(t L[t] \cap A) R$ is a height one prime ideal, so $t a, t b$ cannot be an essential sequence in $R$, so let $b_{1}=t a$ and $b_{2}=t b$.

q.e.d.

It was shown in (5.1) that if $b \notin \cup U(I)$ and $P \in U(I)$, then there exists $Q \in U((I, b) R)$ such that $P \subset Q$. (7.3) shows that this does not hold for $E(I)$.

(7.3) ExAmple. There exists a local domain $R$ such that $R$ has an ideal $I$, an element $b$, and some $P \in E(I)$ such that $b$ is an essential sequence over $I$ and no prime ideal in $E((I, b) R)$ contains $P$.

Proof. Let $R, I, P$, and $b$ be as in the proof of (7.1), so $E((I, b) R)=$ $\{(b, c) R\}$ and $P \not \subset(b, c) R$.

q.e.d.

It was shown in (2.5.7) that $\hat{A}^{*}(I) \cup E(I) \subseteq U(I)$. Our final example, (7.4), shows that this containment may be proper. It also shows that $\operatorname{uecogd}(I)<\operatorname{acogd}(I)$ and uecogd $(I)<\operatorname{ecogd}(I)$ can hold.

(7.4) ExAmple. There exists a local ring $R$ such that $R$ has an ideal $I$ such that $\hat{A}^{*}(I) \cup E(I) \subset U(I)$. 
Proof. Let $(L, N=(a, b, c, d) L)$ be a complete regular local ring of altitude four, let $p=a L, B=\left(a^{2}, b\right) L, K=p \cap B$, and $J=\left(c^{2}, c d\right) L$. Let $P=(a, b) L$ so Ass $L / K=\{p, P\}$. Let $R=L / K, M=N / K, I=(J+K) / K$, $z=p / K$, and $w=P / K$. Then $R$ is a local ring of altitude three, $w$ is an imbedded prime divisor of zero, and $R / w=L / P$ is a complete regular local ring of altitude two such that $c^{\prime}, d^{\prime}$, the $P$-residue classes of $c, d$, are a regular system of parameters. Now $(I+w) / w$ is generated by $c^{\prime 2}, c^{\prime} d^{\prime}$, so $\ell((I+w) / w)=2$. Therefore $M / w \in \hat{A}^{*}((I+w) / w)=U((I+w) / w)$, by $[5$, Theorem 3] and (3.1.1), and so $M \in U(I)$, by (2.5.2). Also, $\operatorname{Rad}(I+w) / w$ $=c^{\prime}(R / w)$ is a principal ideal, so $M / w \notin E((I+w) / w)$, by (2.9). And $\operatorname{Rad}(I+z) / z=(a, c)(R / z)$, so altitude $R / z=3$ implies $M / z \notin E((I+z) / z$, by (2.9). Therefore $M \notin E(I)$, by (2.2.5). Finally, $M / z \notin \hat{A}^{*}((I+z) / z$, by [5, Theorem 3], since $\ell((I+z) / z) \leqslant 2<$ altitude $R / z$ and $R / z$ is quasiunmixed, so $M \notin \hat{A}^{*}(I)$, by (2.2.5).

q.e.d.

\section{REFERENCES}

[1] M. Brodmann, Asymptotic stability of Ass $\left(M / I^{n} M\right)$, Proc. Amer. Math. Soc., 74 (1979), 16-18.

[2] D. Katz, A note on asymptotic prime sequences, Proc. Amer. Math. Soc., 87 (1983), 413-418.

[ 3 ] - Prime divisors, asymptotic $R$-sequences, and unmixed local rings, J. Algebra, 95 (1985), 59-71.

[4] S. McAdam and P. Eakin, The asymptotic Ass, J. Algebra, 61 (1979), 71-81.

[ 5 ] - Asymptotic prime divisors and analytic spreads, Proc. Amer. Math. Soc., 80 (1980), 555-559.

[6] - and L. J. Ratliff, Jr., On the asymptotic cograde of an ideal, J. Algebra, 87 (1984), 36-52.

[ 7 ] — and - Essential sequences, J. Algebra, 95 (1985), 217-235.

[ 8 ] - - Asymptotic Prime Divisors, Springer-Verlag Lecture Notes in Mathematics, No. 1023, Springer-Verlag, New York, N.Y., 1983.

[9] M. Nagata, On the chain problem of prime ideals, Nagoya Math. J., 10 (1956), 51-64.

[10] D. G. Northcott and D. Rees, Reductions of ideals in local rings, Math. Proc. Cambridge Philos. Soc., 50 (1954), 145-158.

[11] L. J. Ratliff, Jr., On quasi-unmixed semi-local ring's and the altitude formula, Amer. J. Math., 87 (1965), 278-284.

[12] - On quasi-unmixed local domains, the altitude formula, and the chain condition for prime ideals (II), Amer. J. Math., 92 (1970), 99-144.

[13] - Locally quasi-unmixed Noetherian rings and ideals of the principal class, Pacific J. Math., 52 (1974), 185-205.

[14] - On Rees localities and $H_{i}$-local rings, Pacific J. Math., 60 (1975), 169-194.

[15] - On the prime divisors of zero in form rings, Pacific J. Math., 70 (1977), 489-517.

[16] - and D. Rush, Notes on ideal covers and associated primes, Pacific J. Math., 73 (1977), 169-191. 
[17] —- On asymptotic prime divisors, Pacific J. Math., 111 (1984), 395-413.

[18] — Asymptotic sequences, J. Algebra, 85 (1983), 337-360.

[19] _ Essential sequences over an ideal and essential cograde, Math. Z., 188 (1985), $383-395$.

[20] - Asymptotic prime divisors and integral extension rings, J. Algebra, 95 (1985), 409-431.

[21] —- Essential sequences and Rees rings, J. Algebra, 99 (1986), 337-354.

[22] D. Rees, A note on form rings and ideals, Mathematika, 4 (1957), 51-60.

[23] D. Whitman, Some remarks on regular local rings, Math. Japonicae, 15 (1970), $15-17$.

\section{Daniel Katz}

Department of Mathematics

University of Kansas

Lawrence, Kansas 66045

Louis J. Ratliff, Jr.

Department of Mathematics

University of California

Riverside, California 92521 